\title{
A Scenario Generator for Evaluating the Social Acceptability of Emerging Technologies
}

\author{
Hannah Meyer, Marion Koelle and Susanne Boll \\ University of Oldenburg, Oldenburg, Germany \\ hannah.meyer@uni-oldenburg.de, marion.koelle@uni-oldenburg.de, \\ susanne.boll@uni-oldenburg.de
}

\begin{abstract}
In addition to functionality, usability and user experience, social acceptability is increasingly recognized as driver (or hindering factor) for the adoption of emerging interface technologies. In consequence, factors influencing social acceptance, the perception of technology usage in presence of other people - both, from the user's and the by-stander's points of view, has become of interest to researchers in Human-Computer-Interaction (HCI). Social acceptance does not only depend on the considered device, but also on design aspects, e.g., input and output modalities, and social context, e.g., usage location or the user's relationship to the bystanders. To investigate these factors, and how they interconnect, prior work made use of scenario visualizations, e.g., photographs, videos, or illustrations, whose creations is often time-consuming and labour-intensive. With SAGE, the Social Acceptability (Scenario) Generator and Evaluator, we present a tool that solves this issue by enabling semi-automatic generation of scenario illustrations for the purpose of evaluating the social acceptability of human-computer interfaces. Embedded into a website, SAGE facilitates evaluation, generation and export (download) of scenarios. Thus, it provides an infrastructure for online and offline scenario evaluation, which contributes to research efforts in the field of social acceptability of emerging technologies and novel interaction paradigms.
\end{abstract}

How to cite this book chapter:

Meyer, H., Koelle, M. and Boll, S. 2020. A Scenario Generator for Evaluating the Social Acceptability of Emerging Technologies. In: Loizides, F., Winckler, M., Chatterjee, U., Abdelnour-Nocera, J. and Parmaxi, A. (eds.) Human Computer Interaction and Emerging Technologies: Adjunct Proceedings from the INTERACT 2019 Workshops. Pp. 101-110. Cardiff: Cardiff University Press. DOI: https://doi.org/10.18573/ book3.1. License: CC-BY 4.0. 


\section{Keywords}

Social acceptability $\cdot$ social acceptance $\cdot$ understanding users $\cdot$ user study methods

\section{Introduction}

With the increasing ubiquity of human-computer interfaces, it becomes increasingly relevant that interface and interactions blend well into social context. As users may be noticed or even observed by other individuals who - consciously or unconsciously - wish to identify their attitudes, goals and intentions, interacting with devices in public can impact on the user's impression management and - in consequence - the social acceptability of the interaction [1]. Consequently, social acceptability in the context of technology usage comprises both, how other people perceive the usage of a technical device and how the user thinks that they do [2]. At the development of emerging technology, social acceptability should be taken into account as a crucial factor and source of potential problems.

\subsection{Using Scenarios to Evaluate Social Acceptability}

Prior work demonstrated that social acceptance of a user's interactions with an interface depends on the usage context and may be influenced by a variety of different factors. These include, for example, the view point [2-3], usage location [4-5], interaction modality [6-7], functionality [8-9] or appearance [10-11] of the device, user type [2,12], audience [4] or usage purpose [3]. There are several options to test the influence of those factors on a user's or their bystanders' attitude towards using a technology. In any case, a study participant must be confronted with the situation to be assessed. In-situ studies may be used [9, 13-14], however, they often do not allow to set and control all variables of interest in the desired way. Thus, the presentation of visualizations of the considered situations, which we refer to as scenarios, has become a valid, and popular alternative.

Videos and Photographs Media which are frequently used to present scenarios are videos and photos. Since many interactions in scenarios include some kind of movement or sound, videos are a well suited presentation format for dynamic scenarios. They are used to investigate, for example, gesture based interaction $[2,4,15-17]$. On the other hand, they require time and effort to create, and the creation of additional videos later-on, e.g., after a study pretest, is prone to introduce confounding variables. In contrast, photographs are easier to administer, and it is easier to create a high number of variations (e.g., scenarios with one to many bystanders). For example, Lum et al. [18] investigated how the perception of humans is affected by the use of technology using 
photos of models wearing various devices. Recently, Schwind et al. [5] explored the social acceptance of virtual reality glasses in various situations, with varying locations, users and bystanders. While both approaches, videos and photos are beneficial to depict scenarios in a rather realistic way, they are also harder to control, and - as they require actors - can potentially introduce racial, cultural or gender bias. Illustrations This issue might be mitigated by using drawn, abstract pictograms - as e.g., proposed in Koelle et al. [3]. This kind of presentations allows to keep environments constant, and increase control over assumptions that are made about depicted persons and also leverages the ability to directly highlight the perspective from which the scenario shall be rated. A similar presentation technique is used by the "moral machine" [19, 20], a website which aims to support the decision making in autonomous cars by understanding social preferences. Pictured individuals are visually characterized by certain features as e.g. age, gender or fitness.

All of these approaches, videos, photos and - in particular - illustrations have the disadvantage that scenario visualizations are time-consuming and labourintensive, in particular if many factors are compared to each other. With our work we provide a more efficient approach that allows to automatically create abstract scenario visualizations based on the independent variables a researcher want to investigate with regard to social acceptability.

\subsection{Contribution Statement}

This paper presents the development of SAGE - short for "Social Acceptability (Scenario) Generator and Evaluator" - an online tool to automatically generate scenario illustrations from various components, i.e. independent variables or constants. Our contributions are two-fold: first, SAGE allows to automatically generate and download customized scenarios for own research purposes. Second, SAGE enables browsing and evaluation of scenarios on the website. In the following, we present the tool's design process, and motivate the decision process as to which components should be included. In addition, we discuss existing questionnaires for the evaluation of social acceptance and motivate our selection. Finally, we will give an overview about the implementation and outline how we intend to evaluate the presented tool as part of our future research.

\section{Concept Development}

While we intentionally designed SAGE to be extensible in terms of components, we selected the components included in this first version based on prior work and expert interviews $(\mathrm{N}=4)$ with researchers working on social acceptability issues with human-computer interfaces. Similarly, while scenarios created with SAGE could be used in conjunction with a variety of existing questionnaires, 
we decided for one set of questions for the current version. Subsequently, we outline and motivate our choices.

\subsection{Selection and Visualization of Scenario Components}

As aforementioned, there is a range of factors found by prior work to be influential on social acceptability. However, there is no indication about their actual relevance for future research. Thus, we conducted expert interviews to create an initial set of components to be included in SAGE. Note, that SAGE is constructed to be extendable - thus, the selected components do not necessarily represent a final choice. All interviewed experts were researchers in the field of Human Computer Interaction who published at least one paper covering social acceptability. Overall, we interviewed four experts (2 female), aged 31 to 42 $(\bar{x}=35, \sigma=5)$ from Europe with 5 to 10 years of research experience in HCI. The experts indicated to have published 2 to 10 papers on social acceptability in HCI.

We conducted semi-structured interviews over Skype that were tied around the topics "Location", "User", "Interaction", "Bystanders" and "General Factors". In addition, the participants were asked to comment on candidate components derived from literature. Notes taken during the interviews were clustered and analyzed for main themes that we present subsequently:

1. Details about the user and the bystanders - such as gender, age, etc. do influence the social acceptance. However, these are not part of interface design. Thus, those factors should be (and have been) investigated detached from technology usage and their examination is therefore not the focus of HCI. We excluded those details from the configuration options provided by SAGE.

2. Both the purpose of use and the interaction modality are exceedingly relevant for social acceptability, the device appearance is considered to play a minor part. This supports our selection of abstract device representations for SAGE. The interaction modality is one of the main components of SAGE.

3. Social context is more relevant for social acceptance than the spatial context. However, locations typically indicate social context and induce the relationships between bystanders and the user. Thus, location is included in SAGE, but could be understood as outline of social context.

From these results, we derived the components and specifications listed in Table 1. We focused on the social acceptability of mobile devices - computing devices small enough to be carried around.

We based the visualization of the components on the bikablo visual dictionaries [21-22] in order to use symbols that are proven to be as understandable as possible. We give an example of one automatically generated scenario in Figure 1. 
Table 1: Scenario Components and their Specifications included in SAGE.

\begin{tabular}{|l|l|}
\hline Component & Specifications \\
\hline Device Kind & $\begin{array}{l}\text { Smart Watch, Smart Phone, Smart Glasses, Smart } \\
\text { Clothing, Electronic Tattoo, Smart Contact Lenses }\end{array}$ \\
\hline Interaction Modality & $\begin{array}{l}\text { None, Speech, Arm Gesture, Hand Gesture, Touch } \\
\text { of Device }\end{array}$ \\
\hline Usage Location & $\begin{array}{l}\text { Neutral, Home, Pavement, Public Transport, } \\
\text { Restaurant, Workplace }\end{array}$ \\
\hline Usage Purpose & $\begin{array}{l}\text { Hidden, Navigation, Entertainment, Information } \\
\text { Access, Assistive Technology, Communication, } \\
\text { Capturing of Memories }\end{array}$ \\
\hline Number of Bystanders & $\begin{array}{l}\text { No Bystander, One Bystander, Two Bystanders, } \\
\text { Many Bystanders }\end{array}$ \\
\hline User-Bystander-Relationship & $\begin{array}{l}\text { Unknown, Partner, Friend, Family, Colleague, } \\
\text { Stranger }\end{array}$ \\
\hline
\end{tabular}

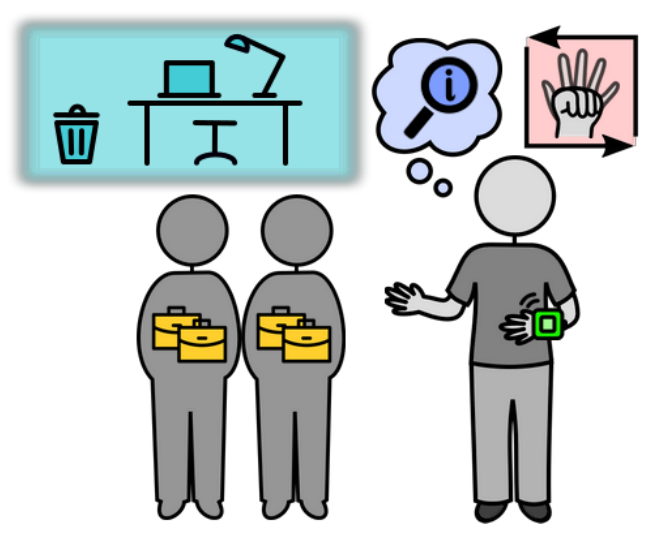

Fig. 1: Exemplary Scenario Created from its Components - A person ("the user") is interacting with his/her smart watch via a hand gesture. He/she does the interaction to get access to information. The scenario takes place at the user's workplace. There are also two bystanders present, who are colleagues of the user.

\subsection{Selection of a Questionnaire}

There is no established social acceptability questionnaire. However, some questions have been (re)used in prior work. These are, for example, the questionnaires proposed by Rico and Brewster $[4,16]$ and by Profita et al. [23]. The latter formulates thirteen statements about the user, the device and their interaction and asks for the participants' degree of agreement to them. The former 
asks the participant to select every location and audience from a list where they would be willing to perform a particular gesture. Some works exactly took over this questionnaire $[7,24]$, others modified the questionnaire for their purposes [6, 25-26]. However, at the SAGE website, location and audience are part of the scenario itself and the interaction modality is not necessarily restricted to gestures only. Thus, for SAGE we propose to adapt the questions to "Are you willing to perform the user's interaction?" or - if the Likert scale items proposed by [26] are adopted - "How willing are you to perform the user's interaction?". Based on prior work, we derived a unified questionnaire, where we aligned the phrasing and response options. This questionnaire, consisting of two items, is part of SAGE's on-site evaluation:

1. How much do you agree to the following statement: 'I would be willing to perform the users interaction in the given context.'?

2. How much do you agree to the following statement:' If I were the bystander,I would rate the user's interaction as acceptable in the given context.?

As response options we chose a 5-point Likert scale adapted from Pearson et al. [27] ranging from "strongly agree" over "agree", "neither agree nor disagree" and "disagree" to "strongly disagree". Doing so, we deliberately give the participant the opportunity to take a neutral position.

\section{Implementation}

The implementation of the SAGE website functionalities was based on the programming language JavaScript due to its applicability as a browser-side as well as a server-side scripting language. To achieve a separation of concerns, we split SAGE into a Vue.js front-end and a Node.js back-end application. The website is currently reachable at the following URL: https://www.sage.uni-oldenburg.de Depending on the parameters of a scenario, SAGE constructs the scenario from a set of sub-images. The tool uses SVG images called by HTML code to
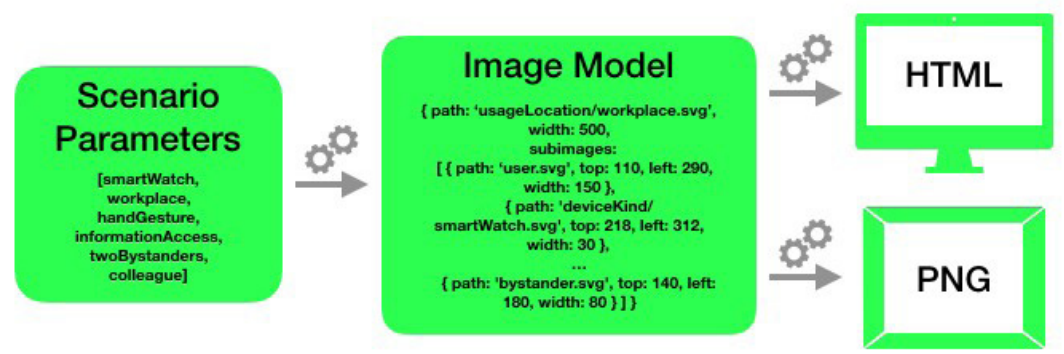

Fig. 2: Overview of SAGE's Image Generation Process. 
display scenarios on the website, but provides downloadable scenario images as PNGs for an improved ease of use. In order to still ensure the same generated image for the same scenario, we established a so-called image model - a detailed description of the image structure for a specific scenario i.e. the necessary subimages with their sizes and positions. The format-independent image model will subsequently be processed into the final scenario image of the requested format. The image generation process is outlined in Figure 2.

\section{Planned Evaluation}

For SAGE to establish itself as a useful tool for research on social acceptability in $\mathrm{HCI}$, it has to facilitate the creation of relevant scenarios for a broad range of research questions. In addition, the tool has to deliver appropriate data for statistical analysis. Based on those requirements, we plan a two-stage evaluation process. First, we aim to collect data directly on the SAGE website and compare the results to results obtained by prior work. For this purpose, we started a data collection in March 2019. In addition, we aim to present SAGE to researchers working on social acceptability aspects of HCI to collect feedback and evaluate its usefulness as a tool.

\section{Conclusion}

In this paper we introduced SAGE, a tool that generates scenarios from components - i.e., independent variables or constants - which facilitates evaluating social acceptability in user studies. Embedded in a website, it allows evaluation, automatic generation as well as export (download) of scenario illustrations for research purposes. Thus, in contrast to the manual creation of scenario illustrations, videos or photos, it increases efficiency, and enhances replicability as well as extendability of study designs. We hope - similar to the notion of "discount usability" [28] - that the fast and easy generation of study materials can promote social acceptability as a field of research by making it easier to get started and eventually prevent technology from failing due to a lack of social acceptance.

\section{References}

1. Goffmann, E.: The Presentation of Self in Everyday Life. In: Contemporary Sociological Theory, pp. 46-74. Wiley (2012)

2. Montero, C., Alexander, J., Marshall, M., Subramanian, S.: Would You Do That? Understanding Social Acceptance of Gestural Interfaces. In: Proceedings of the 12th International Conference on Human-Computer Interaction with Mobile Devices and Services, pp. 275-278. ACM New York, USA (2010) 
3. Koelle, M., Kranz, M., M“oller, A.: Dont look at me that way! Understanding User Attitudes Towards Data Glasses Usage. In: Proceedings of the 17th International Conference on Human-Computer Interaction with Mobile Devices and Services, pp. 362-372. ACM New York, USA (2015)

4. Rico, J., Brewster, S.: Usable Gestures for Mobile Interfaces: Evaluating Social Acceptability. In: Proceedings of the SIGCHI Conference on Human Factors in Computing Systems, pp. 887-896. ACM New York, USA (2010)

5. Schwind, V., Reinhardt, J., Rzayev, R., Henze, N., Wolf, K.: Virtual Reality on the Go? A Study on Social Acceptance of VR Glasses. In: Proceedings of the 20th International Conference on Human Computer Interaction with Mobile Devices and Services. ACM New York, USA (2018)

6. Serrano, M., Ens, B., Irani, P.: Exploring the Use of Hand-To-Face Input for Interacting with Head-Worn Displays. In: Proceedings of the SIGCHI Conference on Human Factors in Computing Systems, pp. 3181-3190. ACM New York, USA (2014)

7. Efthymiou, C., Halvey, M.: Evaluating the Social Acceptability of Voice Based Smartwatch Search. In: Ma, S. et al. (eds.) Information Retrieval Technology. AIRS 2016, LNCS, vol. 9994. Springer, Cham (2016).

8. Choe, E. K., Consolvo, S., Jung, J., Harrison, B., Patel, S., Kientz, J.: Investigating Receptiveness to Sensing and Inference in the Home Using Sensor Proxies. In: Proceedings of the 2012 ACM Conference on Ubiquitous Computing, pp. 61-70. ACM New York, USA (2012)

9. Denning, T., Dehlawi, Z., Kohno, T.: In Situ with Bystanders of Augmented Reality Glasses: Perspectives on Recording and Privacy-Mediating Technologies. In: Proceedings of the SIGCHI Conference on Human Factors in Computing Systems, pp. 2377-2386. ACM New York, USA (2014)

10. Miner, C. S., Chan, D. M., Campbell, C.: Digital Jewelry: Wearable Technology for Everyday Life. In: CHI'01 Extended Abstracts on Human Factors in Computing Systems, pp. 45-46. ACM New York, USA (2001)

11. Shinohara, K., Wobbrock, J. O.: In the Shadow of Misperception: Assistive Technology Use and Social Interactions. In: Proceedings of the SIGCHI Conference on Human Factors in Computing Systems, pp. 705-714. ACM New York, USA (2011)

12. Profita, H. P., Clawson, J., Gilliland, S., Zeagler, C., Starner, T., Budd, J., Do, E. Y.: Don't Mind Me Touching My Wrist: A Case Study of Interacting with On-Body Technology in Public. In: Proceedings of the 2013 International Symposium on Wearable Computers, pp. 89-96. ACM New York, USA (2013)

13. Williamson, J. R., Crossan, A., Brewster, S.: Multimodal Mobile Interactions: Usability Studies in Real World Settings. In: Proceedings of the 13th International Conference on Multimodal Interfaces, pp. 361-368. ACM New York, USA (2011)

14. Hoyle, R., Templeman, R., Armes, S., Anthony, D., Crandall, D., Kapadia, A.: Privacy Behaviors of Lifeloggers using Wearable Cameras. 
In: Proceedings of the 2014 ACM International Joint Conference on Pervasive and Ubiquitous Computing, pp. 571-582. ACM New York, USA (2014)

15. Rico, J., Brewster, S.: Gestures all around us: user differences in social acceptability perceptions of gesture based interfaces. In: Proceedings of the 11th International Conference on Human-Computer Interaction with Mobile Devices and Services. ACM New York, USA (2009)

16. Rico, J., Brewster, S.: Gesture and Voice Prototyping for Early Evaluations of Social Acceptability in Multimodal Interfaces. In: International Conference on Multimodal Interfaces and the Workshop on Machine Learning for Multimodal Interaction. ACM New York, USA (2010)

17. Ronkainen, S., Häkkilä, J., Kaleva, S., Colley, A., Linjama, J.: Tap Input as an Embedded Interaction Method for Mobile Devices. In: Proceedings of the 1st International Conference on Tangible and Embedded Interaction, pp. 263-270. ACM New York, USA (2007)

18. Lum, H. C., Sims, V. K., Chin, M. G., Lagattuta, N. C.: Perceptions of Humans Wearing Technology. In: Proceedings of the Human Factors and Ergonomics Society Annual Meeting, pp. 864-868. SAGE Publishing Los Angeles, USA (2009)

19. Noothigattu, R., Gaikwad, S., Awad, E., Dsouza, S., Rahwan, I., Ravikumar, P., Procaccia, A. D.: A Voting-Based System for Ethical Decision Making. In: Proceedings of the thirty-second AAAI Conference on Artificial Intelligence. AAAI Press (2017)

20. Moral Machine Website, http://moralmachine.mit.edu. Last accessed 26 Apr 2019

21. Scholz, H., Haußmann, M.: bikablo 1: Das Trainerwörterbuch der Bildsprache. 9nd edn. Kommunikationslotsen Much, Germany (2017)

22. Scholz, H., Haußmann, M.: bikablo 2.0: Neue Bilder fur̈ Meeting, Training \& Learning. 7nd edn. Kommunikationslotsen Much, Germany (2014)

23. Profita, H., Albaghli, R., Findlater, L., Jaeger, P., Kane, S. K.: The AT Effect: How Disability Affects the Perceived Social Acceptability of HeadMounted Display Use. In: Proceedings of the 2016 CHI Conference on Human Factors in Computing Systems, pp. 4884-4895. ACM New York, USA (2016)

24. Lv, Z., Halawani, A., Feng, S., Ur Réhman, S., Li, H.: Touch-less Interactive Augmented Reality Game on Vision-Based Wearable Device. In: Personal and Ubiquitous Computing, vol. 19, pp. 551-567. Springer Nature (2015)

25. Hsieh, Y., Jylhä, A., Orso, V., Gamberini, L., Jacucci, G.: Designing a Willing-to-Use-in-Public Hand Gestural Interaction Technique for Smart Glasses. In: Proceedings of the 2016 CHI Conference on Human Factors in Computing Systems, pp. 4203-4215. ACM New York, USA (2016)

26. Bailly, G., Müller, J., Rohs, M., Wigdor, D., Kratz, S.: ShoeSense: A New Perspective on Hand Gestures and Wearable Applications. In: Proceedings 
of the SIGCHI Conference on Human Factors in Computing Systems, pp. 1239-1248. ACM New York, USA (2012)

27. Pearson, J., Robinson, S., Jones, M.: Its About Time: Smartwatches as Public Displays. In: Proceedings of the 33rd Annual ACM Conference on Human Factors in Computing Systems, pp. 1257-1266. ACM New York, USA (2012)

28. Nielsen, J.: Usability Engineering at a Discount. In: Proceedings of the third International Conference on Human-Computer Interaction on Designing and Using Human-Computer Interfaces and Knowledge Based Systems (2nd ed.), pp. 394-401. Elsevier Science Inc. (1989) 